\title{
ADAPTACIÓN DE CULTIVARES DE NOPAL FORRAJERO AL SEMIÁRIDO ESTADO DE PARAÍBA, BRASIL ${ }^{1}$
}

\author{
Aldo Torres-Sales ${ }^{2}$, Mauricio Luiz de Mello Viera Leite ${ }^{3}$, Alberício Pereira de Andrade ${ }^{4}$
}

\section{RESUMEN}

Adaptación de cultivares de nopal forrajero al semiárido estado de Paraíba, Brasil. El objetivo del presente trabajo fue evaluar la adaptación de dos cultivares de nopal forrajero de la especie Nopalea cochenillifera Salm Dyck (Miúda y Alagoas) a las condiciones ambientales del semiárido estado de Paraíba, Brasil. Se utilizó un diseño experimental en bloques al azar con cinco repeticiones. El experimento se realizó en condiciones de campo en el municipio de São João del Cariri, Estado de Paraíba - Brasil (7²2'45,1''S y 36³1'47,2' $\mathrm{W})$, en el periodo de agosto de 2004 a mayo de 2007. Cada veintiocho días se evaluó en la planta la tasa de crecimiento relativo (TCR), en cladodios, la altura, largo, ancho, espesor y número por planta, el área fotosintéticamente activa del cladodio y de la planta. Al final del periodo experimental también se determinó la acumulación de fitomasa por hectárea. A los 1000 días después de la plantación (DDP), los cultivares presentaron tamaños similares para largo y ancho de cladodio. El cultivar Alagoas exhibió cladodios más espesos. El cultivar Miúda presentó mayor número de cladodios $(\mathrm{p}<0,05)$, llegando a producir cuatro veces más que el cultivar Alagoas. Este obtuvo mayor área fotosintéticamente activa de cladodio $(\mathrm{p}<0,05)$; sin embargo, cuando se analizó el área fotosintéticamente activa total de la planta no se constató diferencia entre los cultivares $(p>0,05)$, de la misma forma no se observaron diferencias $(p>0,05)$ en la acumulación de fitomasa. Ambos cultivares presentaron comportamiento similar en las condiciones ambientales del semiárido paraibano.

Palabras-claves: acumulación de biomasa, morfogénesis, producción de forraje, cultivos xerófilos.

\begin{abstract}
Adaptation of spineless cactus to semi-arid region of Paraíba state, Brazil. The aim of this study was to evaluate the adaptation of two spineless cactus of the species Nopalea cochenillifera Salm Dyck (Miuda and Alagoas) under the environmental conditions of the semiarid region in the tate of Paraiba, Brazil. An experimental randomized block design with five replications was used. The experiment was conducted under field conditions in São João do Cariri county, State of Paraiba, Brazil (7²2’45,1"S and $36^{\circ} 31^{\prime} 47,2$ 'W), from August 2004 to May 2007. Every twenty-eight days the relative growth rate of cladodes height, length, width, and thickness were evaluated, as well as the number of cladodes per plant and the photosynthetic active area of cladodes and plant. The biomass accumulation per hectare was also measured at the end of the experimental period. One thousand days after planting, the cultivars did not differ in length and width of cladodes. The Alagoas cultivar showed greater thickness of cladode than the Miuda. The Miuda had a larger number of cladodes $(p>0.05)$. There was no difference ( $p>0.05)$ in biomass accumulation between cultivars. In conclusion, both cultivars showed similar behavior in the environmental conditions of the semiarid region of the State of Paraiba, Brazil.
\end{abstract}

Keyword: biomass accumulation, morphogenesis, forage production, xerophyte crops.

\footnotetext{
Recibido: 24 de febrero, 2015. Aceptado: 18 de junio, 2015. Parte de la tesis para optar por el grado de Ingeniero Zootecnista del primer autor, Universidad Federal da Paraíba - Brasil.

2 Texas Tech University - Lubbock, Texas, USA. aldo.sales@ttu.edu

3 Universidade Federal Rural de Pernambuco - Unidade Acadêmica de Serra Talhada, UFRPE, Caixa Postal 063, 56900-000, Serra Talhada PE - Brasil.nopalea21@yahoo.com.br

4 Universidade Federal da Paraíba - Centro de Ciências Agrárias. Paraíba, Brasil. albericio@uol.com.br
} 


\section{INTRODUCCIÓN}

El futuro de las zonas semiáridas y áridas del mundo depende del desarrollo de sistemas agrícolas sostenibles con cultivos apropiados. Los cultivos en estas áreas deben ser tolerantes al estrés hídrico, las altas temperaturas y la baja fertilidad de los suelos (Lozano, 2011). El nopal atiende la mayoría de estos requisitos, siendo por esto uno de los soportes forrajeros más importantes del Semiárido del noreste brasileño, sobre todo en la región de Cariri paraibano (porción central del semiárido del estado de Paraíba).

En el estado de Paraíba el nopal es la principal fuente de alimentación para los rumiantes, ganando mayor importancia en los periodos de sequía, en especial para las unidades productoras de leche (Sales, 2010).

A pesar de que el número de nopaleras en el semiárido brasileño esté en creciente expansión, casi todos los cultivos son de la especie Opuntia ficusindica Mill (cultivar Gigante y Redonda). En los últimos años con la proliferación de la cochinilla salvaje (Dactylopius opuntiae) ha crecido la atención puesta en los medios de control de esta plaga que viene diezmando los nopales de la región. Dentro de las alternativas de control disponibles, el uso de especies resistentes representa una buena opción, ya que no requiere utilizar productos químicos, contribuyendo a un sistema de producción que proporciona mayor sustentabilidad ambiental (de Vasconcelos et al., 2002).

De las especies resistentes a la cochinilla, la Nopalea cochenillifera Salm Dyck se revela como una opción factible, por ser inmune a este insecto y ser fácilmente encontrada en Brasil, principalmente en los estados de Alagoas, Sergipe, Bahía y Pernambuco (Santos et al., 2007). Es necesario estudiar mejor esta especie, ya que no se dispone de la información correspondiente de cuáles serían las condiciones edafoclimáticas ideales para el cultivo, y además son pocos los estudios que engloban aspectos relacionados con el análisis de crecimiento, ecofisiología y potencial de estas plantas a las diversas condiciones ambientales de Brasil (Albuquerque et al., 2005) .

El análisis de crecimiento es una herramienta para el mejor conocimiento de la planta, pues sus respuestas permiten inferir en actividades fisiológicas, y así estimar las causas de variaciones de crecimiento entre las plantas genéticamente diferentes o entre plantas semejantes creciendo en ambientes diferentes (Lucchessi, 1984).
El objetivo del presente trabajo fue evaluar la adaptación de dos cultivares de Nopal forrajero de la especie Nopalea cochenillifera Salm Dyck (Miúda y Alagoas) a las condiciones ambientales del semiárido del estado de Paraíba - Brasil.

\section{MATERIALES Y MÉTODOS}

El experimento fue realizado en condiciones de campo a partir del mes de agosto de 2005 hasta mayo de 2007, en la Estación Experimental São João de Cariri, base física perteneciente al Centro de Ciencias Agrarias de la Universidad Federal de Paraíba (UFPB), en São João do Cariri - Estado de Paraíba, extremo noreste de Brasil ( $7^{\circ} 22^{\prime} 45,1^{\prime \prime}$ 'S y $36^{\circ} 31^{\prime} 47,2^{\prime \prime}$ 'W) a 500 msnm (Alves, 2013).

El relieve del área experimental es leve ondulado, la insolación media anual es de 2000 a 2900 horas/ año. El clima es del tipo Bsh semiárido, caliente con lluvias de verano/otoño (enero/abril), con temperatura anual promedio de $26{ }^{\circ} \mathrm{C}$, con temperaturas diurnas altas y las noches amenas, con una humedad relativa promedio del aire de 68\% (Koppen (McKnight y Hess, 2000). La precipitación pluvial en el periodo experimental se presenta en la Figura 1.

Antes del plantío, fueron colectadas muestras de suelo representativo del área experimental en las profundidades de 0-20 y 20-40 cm, para su posterior caracterización física y química. El suelo se clasificó como luvisolo crómico-vértico según metodología de Santos et al. (2006). El análisis físico del suelo del área experimental clasificó la textura como franco arenosa. El análisis químico se muestra en el Cuadro 1.

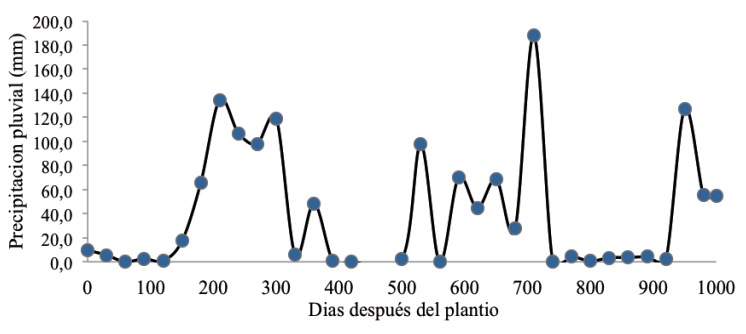

Figura 1. Precipitación pluvial en el periodo experimental en el semiárido paraibano - Brasil (agosto 2005 a mayo 2007).

Figure 1. Rainfall in the experimental period in the semiarid of the State of Paraiba - Brazil (from August 2005 to May 2007). 
Cuadro 1. Análisis químico del suelo del área experimental*. Paraíba, Brasil.

Table 1. Soil chemical analysis of the experimental area*. Paraiba, Brazil.

\begin{tabular}{lcc}
\hline & Determinaciones & Profundidad \\
\cline { 2 - 3 } & $\mathbf{0 - 2 0}$ & $\mathbf{2 0 - 4 0}$ \\
\hline $\mathrm{pH}\left(\mathrm{H}_{2} \mathrm{O}\right)$ & 6,1 & 6,7 \\
$\mathrm{P}\left(\mathrm{mg} / \mathrm{dm}^{3}\right)$ & 1,74 & 0,30 \\
$\mathrm{~K}\left(\mathrm{mg} / \mathrm{dm}^{3}\right)$ & 116,00 & 79,58 \\
$\mathrm{Na}^{+}\left(\mathrm{cmol}_{\mathrm{c}} / \mathrm{dm}^{3}\right)$ & 0,12 & 0,13 \\
$\mathrm{H}^{+}+\mathrm{Al}^{+3}\left(\mathrm{cmol}_{\mathrm{c}} / \mathrm{dm}^{3}\right)$ & 1,16 & 1,00 \\
$\mathrm{Al}^{+3}\left(\mathrm{cmol}_{\mathrm{c}} / \mathrm{dm}^{3}\right)$ & 0,00 & 0,00 \\
$\mathrm{Ca}^{+2}\left(\mathrm{cmol}_{\mathrm{c}} / \mathrm{dm}^{3}\right)$ & 3,90 & 3,70 \\
$\mathrm{Mg}^{+2}\left(\mathrm{cmol}_{\mathrm{c}} / \mathrm{dm}^{3}\right)$ & 4,05 & 2,85 \\
$\mathrm{SB}\left(\mathrm{cmol} / \mathrm{dm}^{3}\right)$ & 8,37 & 6,88 \\
$\mathrm{CTC}\left(\mathrm{cmol}_{\mathrm{c}} / \mathrm{dm}^{3}\right)$ & 9,53 & 7,88 \\
$\mathrm{MO}\left(\mathrm{g} / \mathrm{kg}^{3}\right.$ & 9,63 & 6,19 \\
\hline
\end{tabular}

* Muestra de suelo colectada en agosto de 2005 / Soil sample collected in August 2005.

Se utilizó una densidad de 20000 plantas/hectárea y la especie del nopal forrajero empleado fue la Nopalea cochenillifera. El cultivar Miúda llegó a Brasil en 1932 por importaciones hechas por el gobierno; esta especie es tradicionalmente cultivada en la región noreste del país. El cultivar Alagoas fue obtenido por medio de mejoramiento genético realizado por técnicos de la Secretaria de Agricultura del Estado de Alagoas, resultando como una diploidia cromosómica de la Miúda (Menezes et al., 2005).

Las condiciones, fecha del plantío, control de las malezas, así como los tratos fitosanitarios fueron similares para las variedades examinadas. La fertilización se hizo de acuerdo con los resultados del análisis químico de suelo, se utilizó $60 \mathrm{~kg}$ de N, $40 \mathrm{~kg}$ de $\mathrm{P}_{2} \mathrm{O}_{5}$ e $60 \mathrm{~kg}$ de $\mathrm{K}_{2} \mathrm{O}$. Además, se aplicaron veinte toneladas por hectárea de estiércol caprino, con el fin de mejorar las propiedades del suelo.

Cada veintiocho días se midieron las variables morfométricas: altura de la planta, longitud, ancho, área de los cladodios y espesor. En cada medición se evaluaron todos los cladodios de la planta y se sacó el promedio de cada lectura. Para la determinación del área fotosintética activa, se utilizó la metodología descrita por García de Cortázar y Nobel (1991), donde:
Área fotosintéticamente activa $(\mathrm{AFA})=$ largo del cladodio $(\mathrm{cm}) \mathrm{x}$ ancho del cladodio $(\mathrm{cm}) \mathrm{x}$ 1,32

El área fotosintética total de la planta se obtuvo por la multiplicación de AFA por el número total de cladodios de la planta. Para la determinación de la tasa de crecimiento relativo (TCR), se utilizó la metodología de análisis de crecimiento descrita por Lucchesi (1984), donde:

$$
T C R=\frac{\ln \left(X_{f}\right)-\ln \left(X_{i}\right)}{T}
$$

$X_{f}=$ Variable morfométrica final

$X_{i}=$ Variable morfométrica inicial

$T=$ Intervalo de tiempo entre mediciones

Los valores de grados-días acumulado (GDA) fueron calculados por medio de metodología descrita por Ometto (1981).

El experimento se realizó bajo un diseño en bloques completamente al azar y cinco repeticiones. Cada parcela experimental estuvo constituida por tres hileras de 3,0 m de longitud dando un total de quince plantas por parcela, de las cuales solo fueron analizadas tres plantas cultivadas en la parte central.

Pasados 1000 días después de la plantación, las plantas fueran cortadas y pesadas para la determinación de acumulación de fitomasa por hectárea. Las medias se compararon por prueba $\mathrm{T}$ (de Student) a una significancia de $5 \%$.

\section{RESULTADOS Y DISCUSIÓN}

El acumulado de lluvia fue de $1452,4 \mathrm{~mm}$; la mayor intensidad se observó en los meses de febrero a junio de 2005, sumando un total acumulado de 524 $\mathrm{mm}$ (Figura 1). El periodo de menor intensidad se registró de agosto a diciembre de 2006, con $16 \mathrm{~mm}$. Hubo una gran variación en la intensidad de lluvias a lo largo del periodo experimental. Para Andrade et al. (2006), esta alta variabilidad de las lluvias estimula mecanismos en el metabolismo y adaptación morfológica del nopal específicos y peculiares de cada especie notoriamente en el metabolismo y adaptación morfológica del nopal.

El suelo fue físicamente clasificado como francoarenoso. En el Cuadro 1 se presentan los resultados del 
análisis químico del suelo, demostrando en la camada arable, un $\mathrm{pH}$ de $6,1,8,37 \mathrm{cmol} / \mathrm{dm}^{3}$ para suma de bases, bajo nivel de fósforo $\left(1,74 \mathrm{mg} / \mathrm{dm}^{3}\right)$ y materia orgánica $(9,63 \mathrm{~g} / \mathrm{kg})$. Cabe señalar que el fósforo es un nutriente limitante para el crecimiento del nopal, niveles más bajos que $5 \mathrm{mg} / \mathrm{dm}^{3}$ pueden retardar el crecimiento en hasta 50\% (Nobel, 1989).

La máxima tasa de crecimiento relativo (TCR) (Figura 2) fue próximo a los 200 días después del plantío, en los meses siguientes se observó una evidente disminución en la velocidad de crecimiento en todos los puntos de evaluación. Al comparar los cultivares no se encontraron diferencias $(p>0,05)$ entre ellos a los 1000 DDP. La TCR máxima de los cultivares, de forma general, ocurre durante el periodo inicial de crecimiento, seguido de una disminución gradual hasta la fase de maduración (Pereira y Machado, 1987).

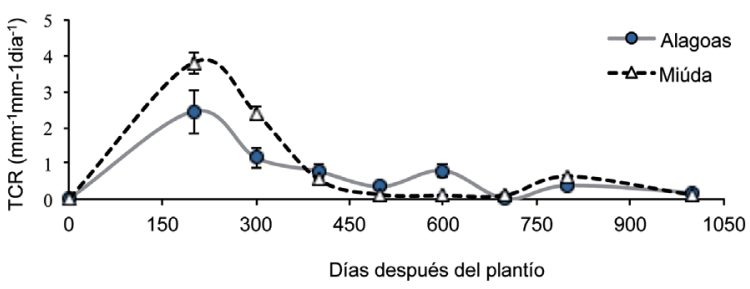

Figura 2. Tasa de crecimiento relativo (TCR) de dos cultivares del nopal de la especie Nopalea cochenillifera hasta los 1000 días después de la plantación (agosto 2005 a mayo 2007), calculada con base en la altura de la planta. Semiárido del estado de Paraíba - Brasil.

Las barras representan la comparación entre promedios a través de la prueba $\mathrm{T}(\mathrm{p}<0,05)$.

Figure 2. Relative growth rate of two spineless cactus cultivars of the species Nopalea cochenillifera until 1000 days after planting (from August 2005 to May 2007), based on plant height. Semi-arid of the State of Paraiba - Brazil.

Bars represent the comparison between averages by means of the $\mathrm{T}$ test $\mathrm{T}(\mathrm{p}<0.05)$.

La TCR para ancho y largo de los cladodios se presenta en la Figura 3, el cultivar Miúda tuvo su máximo crecimiento para esas dos variables entre 150 y 200 días, seguido de una disminución en su velocidad de crecimiento. En el cultivar Alagoas las mayores TCR fueran observadas entre 600 y 800 días después del plantío y un pico de crecimiento expresivo próximo a los 300 días.
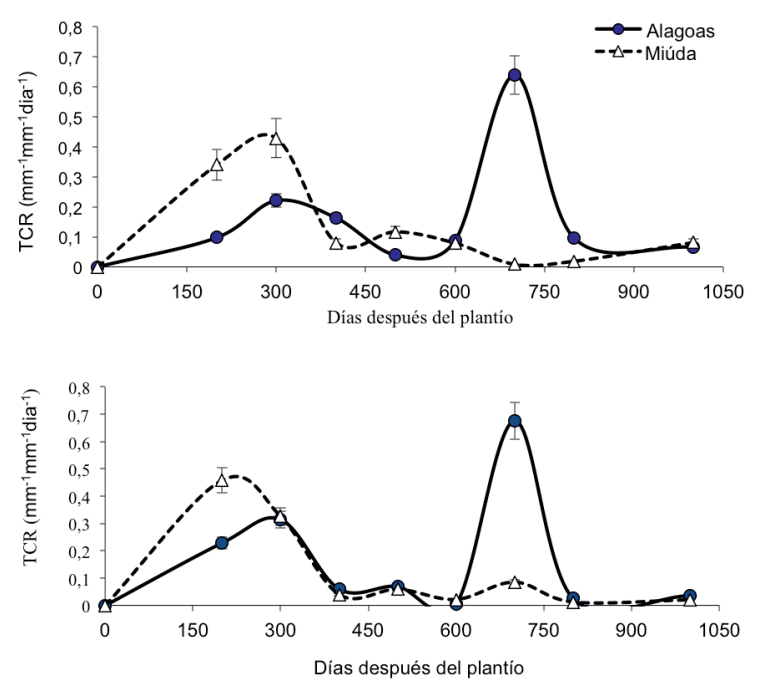

Figura 3. Tasa de crecimiento relativo (TCR) hasta los 1000 días después de la plantación (agosto 2005 a mayo 2007), calculada con base en ancho (A) y longitud (B) de los cladodios de dos cultivares del nopal de la especie Nopalea cochenillifera, en el semiárido del estado de Paraiba - Brasil.

Las barras representan la comparación entre promedios a través de prueba de $\mathrm{T}(\mathrm{p}<0,05)$.

Figure 3. Relative growth rate until 1000 days after planting (from August 2005 to May 2007), based on width (A) and length (B) of cladodes in two cultivars of spineless cactus of the species Nopalea cochenillifera, in the semi-arid of the State of Paraiba - Brazil.

Bars represent the comparison between averages by means of the $\mathrm{T}$ test $\mathrm{T}(\mathrm{p}<0.05)$.

No se observaron diferencias entre los cultivares $(\mathrm{p}<0,05)$ a los 1000 DDP, ya sea para ancho o longitud de cladodio, sin embargo, hubo una gran variación en el crecimiento de estas dos variables. Franco-Salazar y Veliz (2007), al estudiar la influenza del $\mathrm{NaCl}$ en el nopal obtuvieron TCR similares a las que se encontraron en este estudio a una concentración $0 \%$ de $\mathrm{NaCl}$ en el sustrato.

El cultivar Alagoas presentó mayor espesura $(p<0,05)$ de sus cladodios en todos los puntos de evaluación (Figura 4).

Se evidenció una gran variación en la espesura de los cladodios a lo largo de los meses; para Andrade et al. (2006) esto ocurre en función del régimen de lluvias, el cual se encuentra marcado de pulsos e interpulsos de precipitación pluvial. 


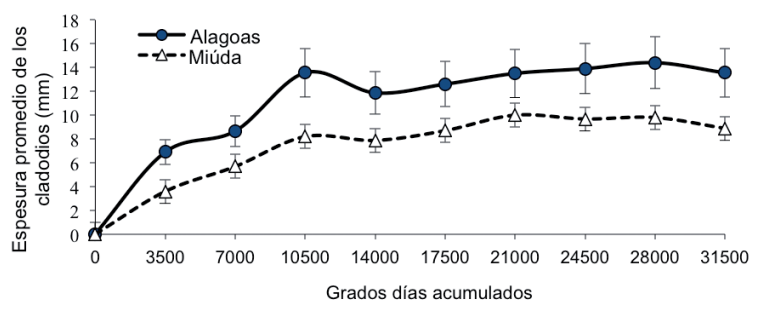

Figura 4. Espesura promedio de los cladodios por planta en función de grados días acumulados (GDA) de dos cultivares del nopal de la especie Nopalea cochenillifera, en el semiárido del estado de Paraíba - Brasil, en el periodo de agosto 2005 a mayo 2007.

Las barras representan la comparación entre promedios a través de la prueba $\mathrm{T}(\mathrm{p}<0,05)$.

Figure 4. Thickness of cladodes per plant according to the accumulated degree-days of two cultivars of spineless cactus of the species Nopalea cochenillifera, in the semi-arid of the State of Paraiba - Brazil, from August 2005 to May 2007. Bars represent the comparison between averages by means of the $\mathrm{T}$ test $(\mathrm{p}<0.05)$.

El cultivar Alagoas presentó cladodios de mayor espesor en comparación con la Miúda, sugiriendo ser una planta con mayor reserva hídrica, por lo tanto, una mayor capacidad de supervivencia en ambientes de baja precipitación pluvial.

Para el número de cladodios por planta (Figura 5), hubo similar tendencia de crecimiento entre los cultivares hasta los 10500 GDA; sin embargo, el cultivar Miúda tuvo un mayor número de cladodios en todos los puntos de evaluación ( $\mathrm{p}<0,05)$, a los 31500 GDA obtuvo cuatro veces más cladodios que el cultivar Alagoas.

El número de cladodios por planta (Figura 5) fue superior a los reportados para la especie Opuntia ficus-indica (Guevara et al., 2000; Zúñiga-Tarango et al., 2009), la cual es ampliamente cultivada en el semiárido del estado de Paraíba; esto denota una gran capacidad de rebrote de esta especie. Cabe señalar que a los 31500 GDA todos los cultivares presentaron cladodios de primer, segundo, tercero y cuarto orden.

En relación al área fotosintéticamente activa de los cladodios (Figura 6) se observó un comportamiento similar en ambos cultivares, con el Alagoas siendo superior $(\mathrm{p}<0,05)$ en todos los puntos de evaluación.

Las superficies fotosintéticas activas encontradas en los cladodios de los cultivares Alagoas y Miúda se muestran en la Figura 6. Según Garcia de Cortazar et al. (2001) valores superiores a $400 \mathrm{~cm}^{2} /$ activo/ $/ \mathrm{cm}^{2} /$ suelo

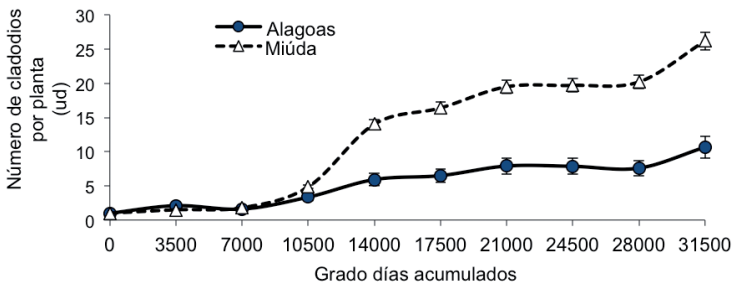

Figura 5. Evolución en el número de cladodios por planta en función de grados días acumulados (GDA) de dos cultivares del nopal de la especie Nopalea cochenillifera, en el estado de Paraiba - Brasil, en el periodo de agosto 2005 a mayo 2007.

Las barras representan la comparación entre promedios a través de la prueba $\mathrm{T}(\mathrm{p}<0,05)$.

Figura 5. Evolution in the number of cladodes per plant according to the accumulated degree-days of two cultivars of spineless cactus of the species Nopalea cochenillifera, in the semi-arid of the State of Paraiba state - Brazil, from August 2005 to May 2007.

Bars represent the comparison between averages by means of the $\mathrm{T}$ test $(\mathrm{p}<0.05)$.

permiten asegurar altas productividades de materia seca para el Nopal. El cultivar Alagoas presentó a los $31500 \mathrm{GDA}$, áreas superiores a $500 \mathrm{~cm}^{2} /$ activo/ $\mathrm{cm}^{2} /$ suelo, mientras que el cultivar Miúda no superó los $300 \mathrm{~m}^{2} /$ activo $/ \mathrm{cm}^{2} /$ suelo. Garcia de Cortazar y Nobel

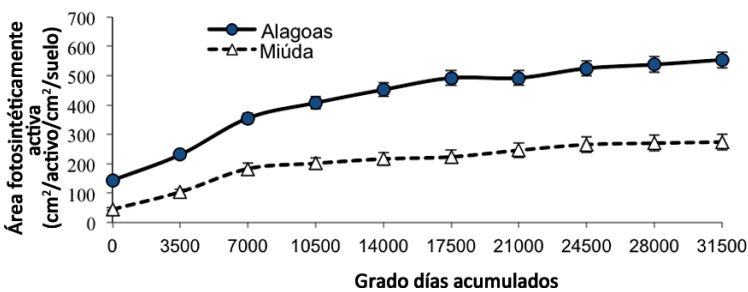

Figura 6. Evolución en el área fotosintéticamente activa de cladodios por planta en función de grados días acumulados de dos cultivares del nopal de la especie Nopalea cochenillifera, en el semiárido paraibano - Brasil, en el periodo de agosto 2005 a mayo 2007.

Las barras representan la comparación entre promedios a través de la prueba $\mathrm{T}(\mathrm{p}<0,05)$.

Figure 6. Evolution of the photosynthetic active area of the cladodes according to the accumulated degreedays of two cultivars of spineless cactus of the species Nopalea cochenillifera, in the semi-arid of the State of Paraiba te - Brazil, from August 2005 to May 2007.

Bars represent the comparison between averages by means of the $\mathrm{T}$ test $(\mathrm{p}<0.05)$. 
(1991) afirman que el comportamiento puede estar en función de las distintas morfologías de los cultivares.

Los valores obtenidos en el cultivar Alagoas en este estudio fueron superiores a lo encontrado por Garcia de Cortazar et al. (2001), quienes estudiaron el efecto de dosis altas de fertilización en la especie Opuntia ficus-indica, reportando valores inferiores a $380 \mathrm{~cm}^{2} /$ activo/ $\mathrm{cm}^{2} /$ suelo.

Para el área fotosintéticamente activa total de la planta (Figura 7) no se observó diferencia estadística $(p>0,05)$ en ambos cultivares; estos mostraron crecimiento linear, fuertemente relacionado con la precipitación pluvial. Aunque el cultivar Miúda obtuvo una menor área de cladodio, al comparar la superficie fotosintéticamente activa total de la planta, no mostró diferencia estadística con el cultivar Alagoas ( $>>0,05)$. Dicho resultado se dio, probablemente, por el elevado número de cladodios por planta.

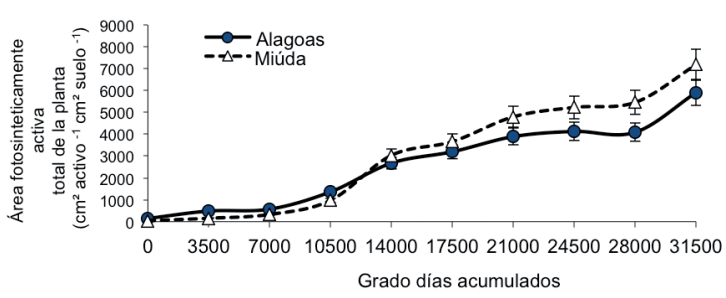

Figura 7. Evolución en el área fotosintéticamente activa total de la planta 1000 días después de la plantación (DAP) de dos cultivares de nopal de la especie Nopalea cochenillifera, en el semiárido paraibano - Brasil en el periodo de agosto 2005 a mayo 2007. Las barras representan la comparación entre promedios por medio de la prueba $\mathrm{T}(\mathrm{P}<0,05)$.

Figure 7. Evolution of the photosynthetic active area of two cultivars of spineless cactus of the species Nopalea cochenillifera 1000 days after planting, in the semi-arid of the State of Paraiba - Brazil, from August 2005 to May 2007.

Bars represent the comparison between average by means of the $\mathrm{T}$ test $(\mathrm{P}<0.05)$.

La acumulación de fitomasa en el periodo experimental se observa en la Figura 8. Esta fue similar para ambos cultivares $(\mathrm{p}>0,05)$, lo que evidencia que la morfología no fue un factor limitante para la acumulación de fitomasa en la especie Nopalea

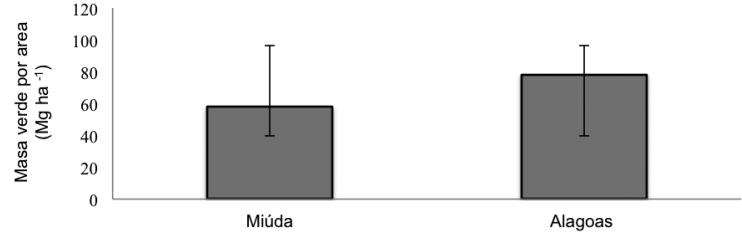

Figura 8. Acumulación de fitomasa (masa verde) por hectare a los 1000 días después de la plantación (DAP) de dos cultivares de palma forrajera de la especie Nopalea cochenillifera, en el semiárido paraibano - Brasil en el periodo de agosto 2005 a mayo 2007. Las barras representan la comparación entre promedios a través de la prueba $\mathrm{T}(\mathrm{P}<0,05)$.

Figure 8. Biomass accumulated per hectare of two cultivars of spineless cactus of the species Nopalea cochenillifera 1000 days after planting, in the semi-arid of the State of Paraiba - Brazil, from August 2005 to May 2007.

Bars represent the comparison between averages by means of the $\mathrm{T}$ test $(\mathrm{P}<0.05)$.

cochenillifera. Este resultado sugiere que en las condiciones ambientales del semiárido paraibano ambos cultivares presentarán una adaptación similar. La disponibilidad de luz, agua y nutrimentos minerales fue suficiente para un pleno desarrollo de las plantas en estas condiciones ambientales (Menezes et al., 2005).

\section{LITERATURA CITADA}

Albuquerque, A., J. Gilson, e J. Santos. 2005. Determinação de fatores da equação universal de perda de solo em Sumé, PB. Rev. Bras. Eng. Agrí. Ambiental 9:153-160.

Alves, J.J.A. 2013. Caatinga do Cariri paraibano. Revista Geonomos 17:19-25.

Andrade, A.P., E.D. Souza, D.S. Silva, I.F. Silva, e J.R Lima. 2006. Produção animal no bioma caatinga: paradigmas dos pulsos-reserva. (En portugués) Rev. Bras. de Zootec. 35:138-155.

de Vasconcelos, A.G.V. de., M. Lira., V. Cavalcanti., M. Santos, e L. Willandino. 2002. Seleção de clones de palma forrageira resistente à cochonilha do carmim (Dactylopius sp.). Rev. Bras. Zoot. 38:827-831.

Franco-Salazar, V.A., and J.A. Veliz. 2007. Respuestas de la tuna Opuntia ficus-indica (L.) Mill. al NaCL. Interciencia 32:125-130. 
Garcia de Cortazar, V., and P.S. Nobel. 1991. Prediction and measurement of high annual productivity for Opuntia ficus-indica. Agric. For. Meteorol. 56:261-272.

Garcia de Cortazar V., M.T. Varnero, and M. Espinosa. 2001. Effect of biofertilizer over photosynthetically active area, cladode production, and nitrogen recovery efficiency in cactus-pear crop (Opuntia ficus-indica L.) on the first year after plantation. J. PACD. 4:93-104.

Guevara, J.C.J., J. Gonnet, and R. Estevez. 2000. Frost hardiness and production of Opuntia forage clones in the Mendoza plain, Argentina. J. Arid Environ. 46:199-207.

Lozano, L. 2011. Cactus pear ecophysiology. Horticultura Argentina 30:37-52.

Lucchesi, A.A. 1984. Utilização prática da análise de crescimento vegetal. An. Esc. Super. Agric. Luiz de Queiroz 41:181-202.

Mcknight, T.L., and D. Hess. 2000. Climate zones and types: the koppen system, physical geography: a landscape appreciation. Upper Saddle River: Prentice Hall, NJ, USA.

Menezes, R.S.C., E.S. Simoes, e E.V.S.B. Sampaio. 2005. A palma no Nordeste do Brasil: conhecimento atual e novas perspectivas de uso. Editora Universitária/ UFPE, Recife, BRA.
Nobel, P.S. 1989. A nutrient index quantifying productivity of agaves and cacti. J. Appl. Ecol. 26: 635-645.

Ometto, J.C. 1981. Bioclimatologia vegetal. Agronômica Ceres. São Paulo-BRA.

Pereira, A.R., e E.C. Machado. 1987. Análise quantitativa do crescimento de comunidade de vegetais. Instituto Agronômico de Campinas. São Paulo, BRA.

Sales, A.T. 2010. Sistemas de producción de nopal forrajero en BRA. RESPYN 5:57-69.

Santos, D., e I. Farias. 2006. Manejo e utilização da palma forrageira (Opuntia e Nopalea) em Pernambuco. (En portugués) Empresa pernambucana de pesquisa agropecuária. Pernambuco, BRA.

Santos, M.V., T. Câmara, e L. Willadino. 2007. Micropropagação de palma forrageira cv. Miúda (Nopalea cochenillifera-Salm Dyck). (En portugués) Rev. Bras. Ciênc. Agr. 2:28-31.

Zúñiga Tarango, R., I. Orona Castillo, C. Vázquez-Vázquez, B. Murillo Amador, E. Zalazar Sosa, J.D. López Martínez, J. García Hernández, y E. Rueda Puente, 2009. Desarrollo radical, rendimiento y concentración mineral en nopal Opuntia ficus-indica (L.) Mill. en diferentes tratamientos de fertilización. J. PACD 11:53-68. 
